\title{
The Mediating Role of Interpretation Bias on the Relationship Between Trait Gratitude and Depressive Symptoms
}

\author{
Anna Alkozei ${ }^{1} \cdot$ Ryan Smith ${ }^{1} \cdot$ Debby L. Waugaman ${ }^{1} \cdot$ Megan D. Kotzin ${ }^{1} \cdot$ \\ Sahil Bajaj ${ }^{1} \cdot$ William D. S. Killgore $^{1}$
}

Accepted: 17 October 2019 / Published online: 30 November 2019

(C) Springer Nature Switzerland AG 2019

\begin{abstract}
High levels of trait gratitude are associated with lower levels of depressive symptoms, but the potential mechanisms underlying this relationship have not been extensively studied. One potential explanation for this relationship is that individuals with higher levels of trait gratitude may have a more positive cognitive style, such that they interpret, attend to, and remember events in a more positive rather than negative manner. This study aimed to explore whether one aspect of a positive cognitive style, a positive interpretation bias, is a mediator in the relationship between trait gratitude and depressive symptoms. During a single visit to the lab, we asked eighty-eight individuals (41 females) to complete a measure of trait gratitude (the Gratitude Resentment and Appreciation Test [GRAT]), two measures of interpretation bias (the Sentence Completion Test for Depression [SCD], and the Ambiguous Scenarios Test for Depression [ASTD]), and a measure of depressive symptoms (the Beck Depression Inventory [BDI-II]). The results of the mediation analyses indicated that a positive interpretation bias partially explained the relationship between trait gratitude and depressive symptoms. These findings suggest that trait gratitude may reduce depressive symptoms in part through its effects on positive thinking patterns. Future longitudinal studies will be needed to elucidate the causal relationship between these variables in greater detail.
\end{abstract}

Keywords Gratitude · Cognitive bias · Depression

The experience of gratitude is familiar to all of us; yet, relative to other constructs (e.g., hope, optimism) it has received less attention in positive psychology research to date (Wood et al. 2010). It has also been a more difficult construct to define consistently

Anna Alkozei

aalkozei@psychiatry.arizona.edu

1 Department of Psychiatry, University of Arizon, 1501 N Campbell Ave, Tucson, AZ 86724, USA 
(reviewed in Wood et al. 2010). However, one generally accepted definition, offered by Wood et al. (2010), describes gratitude as a "life orientation towards noticing and appreciating the positive in the world". In line with this definition, gratitude can be understood to index the cognitive-affective process that occurs when someone explicitly recognizes (i.e., a cognitive process) and appreciates (i.e., an affective process) that something beneficial or otherwise of value was brought into his or her life. Wood et al. (2010) state further that this definition includes the experience of gratitude that occurs toward the source of a benefit, such as recognizing and appreciating the generosity of a specific benefactor (e.g., a friend), but can also involve appreciation of more general sources of benefit occurring outside of one's immediate control (e.g., the weather), as well as experiencing something of value within oneself (e.g., finding the strength to complete a difficult task).

High levels of trait gratitude (i.e., the disposition to experience gratitude more frequently) have been associated with a number of psychological and physical health benefits (see Alkozei et al. 2018 for a review). For example, correlational studies have shown that individuals who report high levels of trait gratitude report greater life satisfaction, happiness, and fewer symptoms of anxiety and depression, as well as better physical health (e.g., less pain) and better sleep quality (Hill et al. 2013; McCullough et al. 2004; Ng and Wong 2013). Longitudinal studies have also shown that higher levels of trait gratitude can predict greater future subjective wellbeing (SWB) in terms of lower depressive symptoms, lower perceived stress, and reduced risk for suicidality one to six months later (Kleiman et al. 2013a, b; Lambert et al. 2012; Wood et al. 2008). These findings suggest a directional relationship whereby higher gratitude leads to lower psychological distress and improved SWB. In addition, intervention studies have also shown that continued 'gratitude practice' (e.g., writing down a list of things for which one is grateful, or writing and delivering a letter expressing gratitude to someone) has beneficial effects on an individual's psychological health (e.g., increased positive emotion, decreased depressive symptoms) and physical health (e.g., better sleep) (Emmons and McCullough 2003; Geraghty et al. 2010a, b; Toepfer et al. 2012). These types of intervention studies have the advantage of being able to show that modifying trait gratitude can lead to changes in psychological and physical health, which further supports the directional relationship proposed in longitudinal studies.

While a growing body of research has replicated the beneficial effects of trait gratitude, as well as the benefits of the practice of gratitude behaviors on psychological health (particularly for reducing negative affect and depressive symptoms), little research has investigated the possible mechanisms explaining this relationship. Alkozei et al. (2018) have recently proposed an evidence-based cognitive model for understanding how gratitude leads to greater SWB (see Fig. 1). In part, they propose that individuals with higher levels of trait gratitude show a more positive cognitive style, in terms of remembering past events in a more positive way (positive memory bias), interpreting negative or ambiguous events more positively (positive interpretation bias), and attending to positive stimuli in the environment more readily (positive attentional bias); they also link these types of cognitive biases to associated neural and physiological changes. These positive cognitive biases then subsequently lead to increased SWB. This model is partly based on the fact that one of the main features of mood disorders, such as depression, is a consistent negative or pessimistic thinking pattern, such that 
ambiguous or even objectively positive situations are automatically interpreted in a negative or threatening way (Amin et al. 1998; Barton et al. 2005; Beck et al. 2002), and that these biases are associated with different functional brain responses (Disner et al. 2011) as well as differences in physiological measures of stress (Gimeno et al. 2009) (for a recent review, see Smith et al. 2018). The presence of a positive cognitive style in highly grateful individuals could therefore explain why gratitude is associated with lower levels of depression and higher levels of positive affect. There is some support for Alkozei et al.'s (2018) model, as Lambert et al. (2012) have shown that the relationship between gratitude and depressive symptoms was, in fact explained by participants' self-reported positive re-framing ability (e.g., seeing challenges as opportunities), a measure of positive interpretation bias. However, the self-report outcome measure used in their study was novel and has not yet been evaluated for its reliability and validity. Lambert et al.'s (2012) proposed mechanism could therefore be tested further by including measurements of cognitive style that do not rely on self-report, such as ambiguous scenarios questionnaires or sentence completion tests that are often used to assess interpretation biases in individuals with psychopathology (Barton et al. 2005; Berna et al. 2011).

Determining the possible mechanisms that underlie the relationship between gratitude and wellbeing is crucial to guide future research. In addition, while interventions to enhance gratitude have been shown to be successful in improving wellbeing in some studies, others have reported only limited success (Kerr et al. 2014). By elucidating the mechanisms underlying the improvements in psychological wellbeing that are brought about by variations in trait gratitude, it may be possible to develop more precise

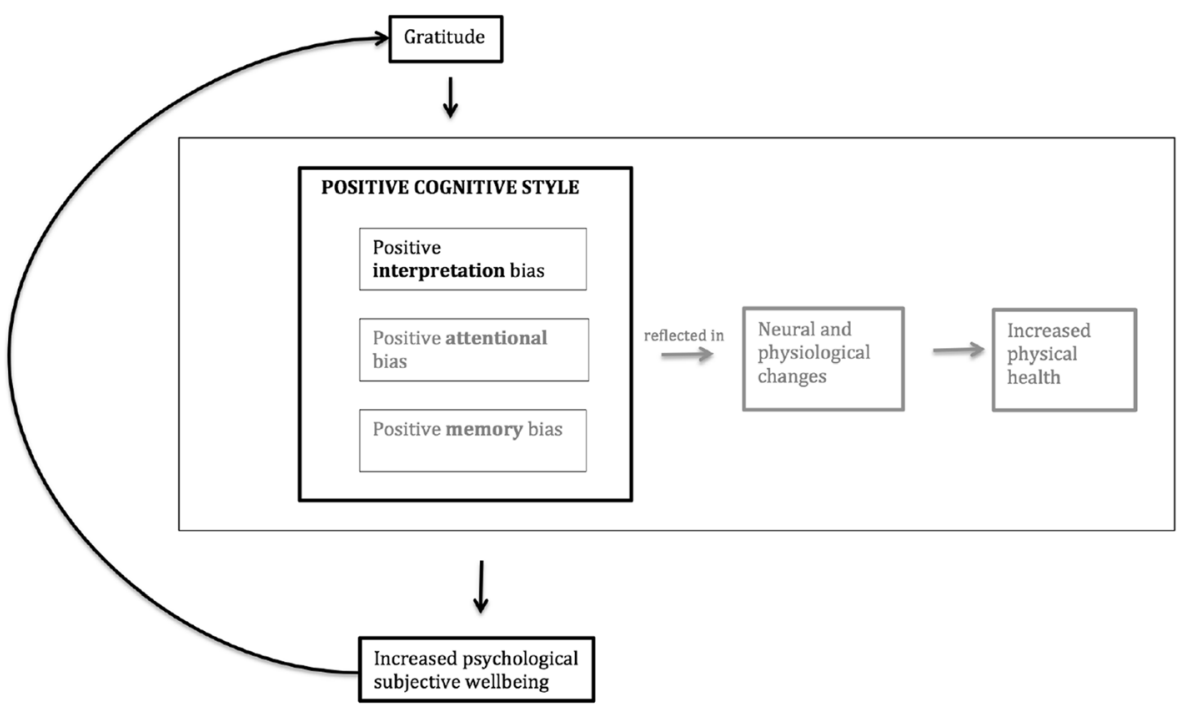

Fig. 1 Cognitive model of gratitude, adapted from Alkozei et al. (2018). The model proposes that gratitude leads to a positive cognitive style, comprised of a positive attentional bias, positive interpretation bias, and positive memory bias. A positive cognitive style is in turn associated with differences in neural and physiological function, both of which lead to better physical health as well as greater psychological wellbeing. Greater psychological wellbeing can then lead to higher levels of gratitude, creating a feedback loop. The current experiment focused on whether a positive interpretation bias could explain the relationship between trait gratitude and depressive symptoms (highlighted in black) 
interventions for treating disorders such as depression, which carry a huge public health burden (Ferrari et al. 2013).

Based on previous experimental and theoretical literature, the aim of the present cross-sectional study was to investigate whether higher levels of trait gratitude are associated with a more positive interpretation bias and whether this may be a significant mediator underlying the known relationship between trait gratitude and depressive symptoms. In particular, this study aimed to extend previous research by using previously validated sentence completion tests and ambiguous scenarios tests as measures of interpretation bias, which are objectively coded for positive, neutral, and negative content (i.e., instead of relying on participant self-report). This cross-sectional study aims to lay the groundwork for future longitudinal investigations using these types of objective measures of cognitive biases.

The specific hypotheses were as follows:

1. Higher levels of trait gratitude will be associated with lower levels of depressive symptoms.

2. Higher levels of trait gratitude will be associated with stronger positive (and weaker negative) interpretation biases on objective (i.e., non-self-report-based) measures.

3. Differences in positive/negative interpretation biases will mediate the relationship between trait gratitude levels and depressive symptom levels.

\section{Methods}

\subsection{Participants}

For this cross-sectional study, eighty-eight participants (41 females) between 18 and 29 years of age $(\mathrm{M}=19.06, \mathrm{SD}=1.85)$ took part in the present study. The sample was predominantly Caucasian $(72.72 \%)$, but also included individuals identifying as Asian (11.36\%), African American (4.45\%), Native American (2.27\%), or 'Other' (9.09\%). In addition, $11.36 \%$ identified as having Hispanic/Latino ethnicity in addition to their race. All participants provided written informed consent prior to taking part in this study. All participants were undergraduate students enrolled in an introductory psychology class at a large state university in the Southwestern U.S. Participants signed up for the study online and received partial course credit for participation. The study protocol was approved by the Institutional Review Board of the University of Arizona.

\subsection{Measures}

Gratitude Resentment and Appreciation Test (GRAT) The GRAT (Watkins et al. 2003) is a 44-item questionnaire measuring trait gratitude in terms of appreciation for life's simple pleasures (e.g., "I think that it's important to 'stop and smell the roses'), their sense of abundance (e.g., "Part of really enjoying something good is being thankful for that thing"), and their social appreciation (e.g., "I couldn't have gotten where I am today without the help of many people"). Participants indicate their agreement with 
each of these statements on 9-point Likert scale (ranging from "I strongly disagree with this statement" to "I strongly agree with this statement"). As suggested by Watkins et al. (2003), total scores on the GRAT were calculated, with higher scores indicating higher levels of trait gratitude. The GRAT has been shown to have good internal consistency, factorial validity, construct validity and temporal stability (Watkins et al. 2003). Internal reliability for the current sample was good $(\alpha=.81)$.

Beck Depression Inventory-Second Edition (BDI-II) The BDI-II (Beck et al. 1996) is a 21-item self-report questionnaire to assess depressive symptoms experienced over the preceding two weeks. Individuals are asked to pick one of four statements for each item (e.g., Item 1. Sadness: "I do not feel sad.", "I feel sad much of the time", "I am sad all of the time", "I am so sad or unhappy that I can't stand it"). According to the standard scoring procedures, total scores are derived by then summing the point values assigned to each chosen statement across the 21 items. Total scores from 0 to 10 are considered normal, 11-16 indicate mild mood disturbance, 17-20 are considered borderline clinical depression, 21-30 indicate moderate depression and scores above 30 are considered severe depression. The BDI-II has been shown to have good internal consistency, and validity among university students (Storch et al. 2004). Internal reliability for the current sample was excellent $(\alpha=.92)$.

Sentence Completion Test for Depression (SCD) The SCD (Barton and Morley 1999) is a measure of an individual's tendency to automatically think positive or negative thoughts in response to abstract or generic cues. Participants are presented with 48 incomplete sentences and asked to complete each sentence in their own words with the first thought that comes to mind. The incomplete sentences include 32 agent-verb combinations (e.g., "I think...", "He could not...") and 16 simple nouns (e.g., "The future...", "The world..."). The agent-verb combinations are presented in a self-agent form (e.g., "I love...") as well as in other-person agent forms (e.g., "Other people enjoy..."). The simple noun combinations include statements about the world (e.g., "Our society..."), the future (e.g., "Next week..."), the past (e.g., "Last year..."), and interpersonal relationships (e.g., "My mother...").

The completed sentences were coded in line with the coding manual of Barton et al. (2005). Detailed information about the coding guidelines is described in Barton et al. (2005). In brief, sentences were coded as positive, negative, or neutral. In order to ensure context of meaning, the whole sentence was classified, and not just the portion completed by the participant. Sentences were coded as positive if the sentence expressed satisfaction, contentment, pleasurable emotions, harmonious relationships with others, or optimism about plans or goals. Sentences were coded as negative if the sentence expressed distressing, painful or unpleasant emotions, inharmonious relationships or pessimism about plans or goals. Statements were coded as neutral if they contained factual information with no positive or negative content, or a combination of both. The first author, blind to participants' gratitude and BDI scores, coded all complete sentences. A second independent coder, also blind to participants' gratitude and depression scores, coded a subset of the sentences $(n=20)$. Inter-rater reliability was good for both negative $(\mathrm{ICC}=.85)$ and positive statements $(\mathrm{ICC}=.85)$. Positive interpretation bias is quantified for this measure by summing the number of positive sentence completions (SCD+), and negative interpretation bias is quantified by 
summing the number of negative sentence completions (SCD-). The SCD has been shown to have good construct validity and cognitive specificity (Barton and Morley 1999).

Ambiguous Scenarios Test for Depression (ASTD) The ASTD (Berna et al. 2011) is another measure previously developed to assess a negative interpretation bias in individuals with depression. The ASTD contains 23 situations that were deliberately ambiguous regarding their valence (e.g., "You wake up, get out of bed, stretch and really notice how you feel today."). Participants are instructed to form a mental image of the scenario happening to them personally, follow the first image that comes to mind, and write down a description of the image. Responses were coded as positive, negative, or neutral using the same guidelines as for the SCD. Positive interpretation bias on the ASTD is quantified by summing the number of positive responses (ASTD+), whereas negative interpretation bias is quantified by summing the number of negative responses (ASTD-). The first author blind-coded all responses, and an independent rater blindcoded a subset of the sentences $(n=20)$. Inter-rater reliability was good for both negative $(\mathrm{ICC}=.95)$ and positive statements $(\mathrm{ICC}=.92)$.

\subsubsection{Procedure}

Participants were tested individually in a laboratory testing room with a table and two chairs in the Department of Psychiatry at the University of Arizona; they completed all questionnaires and tasks on a laptop computer. Additionally, participants completed several other questionnaires not under investigation in the present paper. All of the questionnaires were completed in the same order, but two alternative versions of the SCD and ASTD were used, such that the order of the items was reversed across the two forms, and participants were counterbalanced to receive one of the two versions. This was done in order to prevent that all participants gave detailed responses to the first items and brief answers to the last items. After completion of the questionnaires, participants were debriefed, and thanked for their participation.

\subsubsection{Statistical Analysis}

Complete datasets were present for the whole sample. The first hypothesis was tested using a Pearson's correlation, correlating trait gratitude (GRAT scores) with depressive symptoms (BDI-II scores). The second hypothesis was also tested using a Pearson's correlation, correlating GRAT scores with each of the four interpretation bias scores (i.e., SCD+ and SCD- and ASTD+ and ASTD- scores).

The third hypothesis was tested using Hayes' PROCESS tool in SPSS (http://www. processmacro.org). In order to explore the hypothesis that interpretation bias mediates the relationship between gratitude and depressive symptoms, mediation analyses using Hayes' PROCESS tool in SPSS were conducted for SCD and ASTD positive and negative interpretation bias scores separately. GRAT scores were entered as the independent variable, and SCD+, SCD-, ASTD+ and ASTD- scores were entered as the potential mediators (i.e., each separately), and BDI-II scores were entered as the outcome variable. While PROCESS can test many different types of models, simple mediation analysis is represented by model 4 with 1000 bootstrap samples, which was used for the present analysis. PROCESS uses a regression-based path-analytic 
framework and estimates the total effect (i.e., the effect of the independent variable on the outcome), the direct effect (i.e., the effect of the independent variable on the outcome after accounting for the effects of the mediator), the indirect effect (i.e., the effect of the mediator on the relationship between the independent and outcome variable), and bias-corrected confidence intervals (CI). An indirect effect is considered significant if the CI does not contain zero, which suggests that the mediator explains a significant proportion of the variance of the relationship between the independent variable and the outcome variable. However, if the direct effect remains significant, this highlights that this single mediator can explain some, but not all, of the shared variance between the independent variable and the outcome variable.

\section{Results}

\subsection{Descriptive Statistics}

Participants' descriptive statistics are reported in Table 1.

\subsection{Associations Between Gratitude, Depressive Symptoms, and Interpretation Bias}

Higher levels of trait gratitude, as measured with the GRAT, were associated with lower scores on the BDI-II $(r=-.55, p<.001)$, and higher SCD+ $(r=.44, p<.001)$ and ASTD+ scores $(r=.26, p=.01)$, and lower ASTD- scores $(r=-.33, p<.001)$. No association was found for GRAT scores and SCD- scores $(r=-.009, p=.93)$. There was a positive, but weak, relationship between SCD+ and ASTD+ scores $(r=.21$, $p=.05)$, but no association between the SCD- and ASTD- scores $(r=.03, p=.80)$.

\subsection{Mediation Analyses}

There was a significant indirect effect of SCD+ scores on the relationship between GRAT scores and BDI-II scores $(b=-.05,95 \%$ CI $[-.08,-.02])$, which represents a medium effect size $\left(\mathrm{K}^{2}=.20,95 \%\right.$ CI $\left.[.09, .31]\right)$. In addition, there was also a significant indirect effect of ASTD+ scores on the relationship between GRAT scores

Table 1 Descriptive statistics of all study measures

\begin{tabular}{lllll}
\hline & $\begin{array}{l}\text { Mean (SD) } \\
N=88\end{array}$ & Minimum-maximum & Skewness & Kurtosis \\
\hline GRAT & $308.77(42.55)$ & $209-387$ & -.28 & -.56 \\
BDI-II & $12.72(10.61)$ & $0-54$ & 1.57 & 2.88 \\
SCD positive sentence completions & $16.20(4.15)$ & $6-26$ & -.23 & -.07 \\
SCD negative sentence completions & $13.20(4.10)$ & $6-28$ & 1.16 & 2.25 \\
ASTD positive responses & $9.10(3.34)$ & $1-20$ & .20 & .26 \\
ASTD negative responses & $4.53(3.27)$ & $0-16$ & 1.09 & $.25=6$ \\
\hline
\end{tabular}

GRAT Gratitude Resentment and Appreciation Test, BDI-II Beck Depression Inventory, SCD Sentence Completion Test for Depression, ASTD Ambiguous Scenarios Test for Depression 
and BDI-II scores $(\mathrm{b}=-.01,95 \% \mathrm{CI}[-.03,-.001])$ albeit with a small effect size $\left(\mathrm{K}^{2}=.05\right.$, CI $\left.[.006, .12]\right]$. Finally, there was a further significant indirect effect of ASTD- scores on the relationship between GRAT scores and BDI-II scores $(b=-.03$, CI[-.06, -.01]), with a medium effect size $\left(\kappa^{2}=.14\right.$, CI $\left.[.06, .25]\right)$ (see Fig. 2). These findings suggest that the relationship between higher levels of trait gratitude and lower levels of depressive symptoms may be explained in part by a stronger positive interpretation bias (using both the SCD and ASTD as measures of positive interpretation bias) and a weaker negative interpretation bias (as measured on the ASTD) in those with higher levels of trait gratitude.

\section{Discussion}

In this study, we found evidence that individuals with higher levels of trait gratitude have a more positive interpretation bias, and that this may explain why such individuals also display lower levels of depressive symptoms. This replicates and builds on previous research, which has also shown positive associations between trait gratitude and levels of happiness, life satisfaction, optimism, and physical health, including better sleep quality and greater energy levels (see Alkozei et al. 2018 for a review). Our findings here suggest that individuals with higher levels of trait gratitude also think more positively about themselves, other people, and the world around them generally, and that they may have a stronger expectation for positive (rather than negative) outcomes in uncertain situations.

One way of understanding these findings may be to highlight the fact that, in order to experience high levels of gratitude, an individual needs to (1) be cognizant of when and why some object of need or value was received, and (2) appreciate the value of that object - in many cases recognizing that the receipt of this object was the result of someone else's generosity or provided by another source outside of the receiving individual's control. Individuals with high levels of trait gratitude may therefore engage more regularly in cognitively recognizing and appreciating the positive in the world in a more general sense. Repeatedly engaging in this cognitive routine might ultimately lead to an automatic positive thinking style about themselves, other people, and the world, leading to lower levels of depressive symptoms.

It is worth noting that our two measures of interpretation bias (the SCD and the ASTD) did not correlate highly with one another, and that a negative interpretation bias on the ASTD, but not on the SCD, mediated the relationship between trait gratitude and depressive symptoms. It therefore appears that, while both scales measure a form of interpretation bias, they may nonetheless measure somewhat different aspects of an individual's thinking patterns. We suggest that the SCD, which asks individuals to complete short sentence stems (e.g., "I am...", "The future...", “Other people...") more

Fig. 2 Results of the significant mediation analyses between trait gratitude (GRAT) and depressive symptoms (BDI-II) scores, examining each of the following mediators: (a) SCD+ scores, (b) ASTD+ scores and (c) ASTD- scores. The figures show the total effect, direct effect, and indirect effect of each mediation analysis as well as beta coefficients of each individual pathway of the model. GRAT: Gratitude, Resentment and Appreciation Test, BDI-II: Beck Depression Inventory, SCD: Sentence Completion Test for Depression, ASTD: Ambiguous Scenarios Test for Depression 
(a) Positive cognitive style ( $\mathrm{SCD}+)$

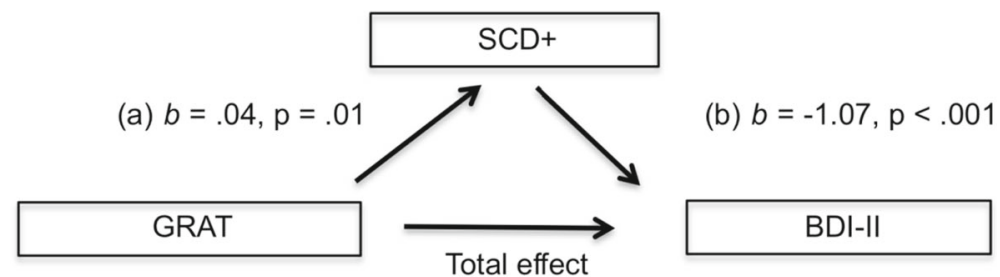

(c) $b=-.14, p<.001$

Direct effect

(c') $b=-.09, p<.001$

Indirect effect

(ab) $b=-.05,95 \% \mathrm{Cl}[-.08,-.02]$

(b) Positive cognitive style (ASTD+)

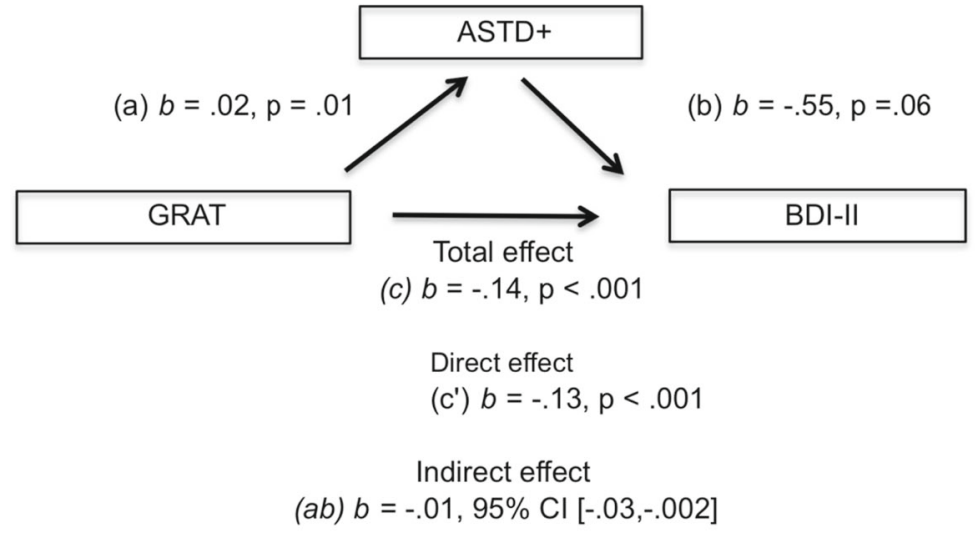

(c) Negative cognitive style (ASTD-)

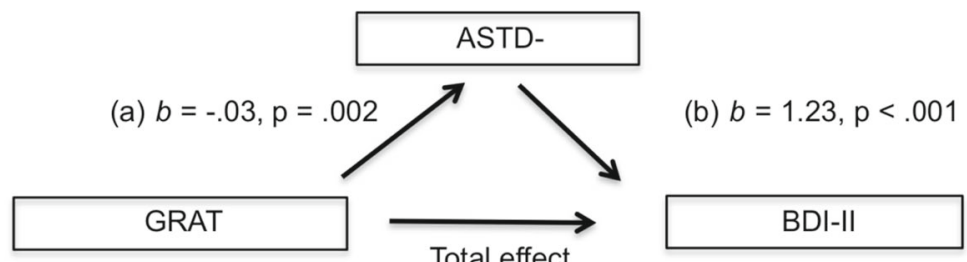

(c) $b=-.14, p<.001$

Direct effect

(c') $b=-.11, p<.001$

Indirect effect

(ab) $b=-.03,95 \% \mathrm{Cl}[-.06,-.01]$ 
plausibly taps into very broad and stable thinking patterns, such as beliefs about oneself, other people, the future, and the past. These types of stable beliefs may pertain most to the broad or abstract "cognitive schemas" (e.g., "I am worthless") described in cognitive models of depression (Beck et al. 2002). The ASTD, on the other hand, asks individuals about the possible outcomes of more specific scenarios; it may therefore be more closely linked to the propensity to experience "automatic negative thoughts" in particular situations - a separate construct that is also described in cognitive models of depression (Beck et al. 2002). These results therefore highlight the importance of understanding how these two measures may account for different aspects of cognitive symptoms of depression, as well as the importance of understanding how gratitude might differentially impact these two aspects of interpretation bias. Such questions represent important topics for future research in this area.

While our findings are correlational in nature, they are supported by previous causal studies that have measured other aspects of a positive cognitive style. For example, Watkins et al. (2015) found that, after a 1-week gratitude intervention, individuals reported a more positive memory bias (i.e., one aspect of a positive cognitive style) and reported greater SWB than individuals in a control condition. In addition, Lambert et al. (2012) found that changes in individuals' self-reported ability to positively reframe negative situations after a gratitude intervention was a significant mediator in the relationship between trait gratitude and depressive symptoms. The results from the present study therefore support and complement these previous findings. More specifically, the major contribution of the present results is that (1) they focus on a different aspect of positive cognitive style than did Watkins and colleagues (i.e., interpretation bias vs. memory bias), and (2) our measures of interpretation bias did not rely on selfreport (i.e., unlike the findings of Lambert and colleagues), and therefore provide a more objective and validated approach to confirming the relationships between each of these interesting variables.

In addition, the results support Alkozei et al.'s (2018) cognitive model of gratitude, which proposes that gratitude leads to a positive cognitive style (i.e., indexed by a positive interpretation bias, a positive memory bias, and a positive attention bias), which in turn leads to greater psychological and physical well-being. Alkozei et al. (2018) also propose that such a positive cognitive style may have detectable neural and physiological correlates. However, future research will be necessary to establish whether a positive cognitive style in individuals with higher levels of trait gratitude is in fact associated with individual differences in neural and physiological functioning, and whether these differences may in turn contribute to better physical health outcomes.

It should be noted that the direct effect of trait gratitude on depressive symptoms remained significant after accounting for positive interpretation biases, suggesting that a positive interpretation bias can only partially explain the relationship between trait gratitude and depressive symptoms. It can therefore be assumed that other factors likely also play a role; identifying these additional factors remains an important topic for future research as well. For example, it has been shown that higher levels of both trait and state gratitude are associated with (1) greater self-reported social support, (2) greater reciprocal and upstream altruism (i.e., passing benefits to the benefactor as well as a third party) (Bartlett and DeSteno 2006; Wood et al. 2008), and (3) higher levels of relationship satisfaction and connection (Algoe et al. 2010; Chang et al. 2013). Low levels of social support have in turn been shown to be associated with poorer 
psychological health, including depression (Peirce et al. 2000). It has therefore been proposed that social support may be another mediating factor contributing to the relationship between gratitude and psychological health, and that interventions involving behavioral expression of gratitude may contribute to improved health status by enhancing social support (Alkozei et al. 2018); however this has not yet been directly tested. Future studies are therefore needed to investigate the possible relative contributions of cognitive biases and social support in explaining these relationships in greater detail.

\subsection{Strengths and Limitations}

While our study benefits from using objective measures of interpretation bias, rather than self-report (as was used in previous studies), it should be noted that the directionality of our observed effects remains speculative due to the cross-sectional design of our study. While we suggest one directional interpretation (based on previous research and theory), our data remains correlational in nature, and it is therefore impossible to determine with confidence whether (1) individuals who report higher levels of trait gratitude develop a more positive interpretation bias and as a result experience lower levels of depressive symptoms, or (2) whether these relationships are better explained through alternative causal pathways. Future studies should make use of a longitudinal experimental design in order to examine whether increases in trait gratitude (e.g., after a gratitude intervention) in fact lead to decreases in depressive symptoms, and whether this effect is mediated by associated changes in interpretation biases.

Another limitation worth mentioning is that this study was conducted in a relatively small group of undergraduate students enrolled in an introductory psychology class; therefore, the generalizability of these findings to individuals of other ages and backgrounds remains open for future investigation. Lastly, clinical depression was not assessed, and whether these findings apply to individuals with a clinical diagnosis of major depressive disorder (rather than the sub-clinical levels of depressive symptoms examined here) is unclear and should also be the focus of future investigations.

\subsection{Implications for Future Research/Clinical Practice}

Gratitude interventions have been increasingly studied, but the exact mechanisms that account for why they lead to increases in psychological wellbeing have not yet been established. Based on our findings, and the other findings we have reviewed, it appears plausible that undergoing a gratitude intervention may lead to a more positive cognitive style; this is because individuals in such interventions are effectively asked to "practice" reflecting on the positive aspects of life that were brought about by other people or external sources. This "practice," when repeatedly reinforced, may then result in a change in one's cognitive habits more generally (e.g., thinking about oneself, other people, and the world in a more positive way), which could in turn lead to improved affect over time.

Our results regarding weaker negative interpretation biases in more grateful individuals may also suggest ways of improving gratitude interventions. For example, they suggest that it may be beneficial to specifically ask individuals to think about negative or neutral events they are grateful for during such interventions; if such instructions 
were added, it could perhaps better train the ability to interpret neutral or ambiguous events in a more positive way (and therefore weaken negative interpretation biases). This is an open research question for future investigations, but our study may help to lay the groundwork for such future longitudinal intervention studies.

\section{Conclusion}

Higher levels of trait gratitude were associated with lower depressive symptoms, and this relationship could be partly explained by the tendency of more grateful individuals to interpret ambiguous cues in a more positive manner. Gratitude interventions may therefore be effective because they require individuals to practice positive thinking habits (i.e., frequently engaging in the explicit recognition of, and appreciation for, the value of their possessions, circumstances, experiences, and relationships), which would in turn plausibly contribute to increased happiness and decreased negative affect. However, future longitudinal research is necessary to disentangle the causal relationships between these variables in greater detail, and to investigate what other kinds of mechanisms are also able to partially explain the relationship between gratitude and depressive symptoms.

\section{References}

Algoe, S. B., Gable, S. L., \& Maisel, N. C. (2010). It's the little things: Everyday gratitude as a booster shot for romantic relationships. Personal Relationships, 17(2), 217-233.

Alkozei, A., Smith, R., \& Killgore, W. D. (2018). Gratitude and subjective wellbeing: A proposal of two causal frameworks. Journal of Happiness Studies, 19(5), 1519-1542.

Amin, N., Foa, E. B., \& Coles, M. E. (1998). Negative interpretation bias in social phobia. Behaviour Research and Therapy, 36(10), 945-957.

Bartlett, M. Y., \& DeSteno, D. (2006). Gratitude and prosocial behavior helping when it costs you. Psychological Science, 17(4), 319-325.

Barton, S. B., \& Morley, S. (1999). Specificity of reference patterns in depressive thinking: Agency and object roles in self-representation. Journal of Abnormal Psychology, 108(4), 655.

Barton, S. B., Morley, S., Bloxham, G., Kitson, C., \& Platts, S. (2005). Sentence completion test for depression (SCD): An idiographic measure of depressive thinking. British Journal of Clinical Psychology, 44(1), 29-46.

Beck, A. T., Steer, R. A., \& Brown, G. K. (1996). Beck depression inventory-II. San Antonio.

Beck, A. T., Dowd, E., \& Leahy, R. (2002). Cognitive models of depression. Clinical Advances in Cognitive Psychotherapy: Theory and Application, 14, 29-61.

Berna, C., Lang, T. J., Goodwin, G. M., \& Holmes, E. A. (2011). Developing a measure of interpretation bias for depressed mood: An ambiguous scenarios test. Personality and Individual Differences, 51(3), 349354.

Chang, Y.-P., Li, T.-S., Teng, H. Y., Berki, A., \& Chen, L. H. (2013). Living with gratitude: Spouse's gratitude on one's depression. Journal of Happiness Studies, 14(4), 1431-1442.

Disner, S. G., Beevers, C. G., Haigh, E. A., \& Beck, A. T. (2011). Neural mechanisms of the cognitive model of depression. Nature Reviews Neuroscience, 12(8), 467-477.

Emmons, R. A., \& McCullough, M. E. (2003). Counting blessings versus burdens: An experimental investigation of gratitude and subjective well-being in daily life. Journal of Personality and Social Psychology, 84(2), 377.

Ferrari, A. J., Charlson, F. J., Norman, R. E., Patten, S. B., Freedman, G., Murray, C. J., et al. (2013). Burden of depressive disorders by country, sex, age, and year: findings from the global burden of disease study. PLoS Medicine, 10(11), e1001547. 
Geraghty, A. W., Wood, A. M., \& Hyland, M. E. (2010a). Attrition from self-directed interventions: Investigating the relationship between psychological predictors, intervention content and dropout from a body dissatisfaction intervention. Social Science \& Medicine, 71(1), 30-37.

Geraghty, A. W., Wood, A. M., \& Hyland, M. E. (2010b). Dissociating the facets of hope: Agency and pathways predict dropout from unguided self-help therapy in opposite directions. Journal of Research in Personality, 44(1), 155-158.

Gimeno, D., Kivimäki, M., Brunner, E. J., Elovainio, M., De Vogli, R., Steptoe, A., . . Marmot, M. G. (2009). Associations of C-reactive protein and interleukin-6 with cognitive symptoms of depression: 12-year follow-up of the Whitehall II study. Psychological Medicine, 39(3), 413-423.

Hill, P. L., Allemand, M., \& Roberts, B. W. (2013). Examining the pathways between gratitude and self-rated physical health across adulthood. Personality and Individual Differences, 54(1), 92-96.

Kerr, S. L., O’Donovan, A., \& Pepping, C. A. (2014). Can gratitude and kindness interventions enhance wellbeing in a clinical sample? Journal of Happiness Studies, 16(1), 17-36.

Kleiman, E. M., Adams, L. M., Kashdan, T. B., \& Riskind, J. H. (2013a). Grateful individuals are not suicidal: Buffering risks associated with hopelessness and depressive symptoms. Personality and Individual Differences, 55(5), 595-599.

Kleiman, E. M., Adams, L. M., Kashdan, T. B., \& Riskind, J. H. (2013b). Gratitude and grit indirectly reduce risk of suicidal ideations by enhancing meaning in life: Evidence for a mediated moderation model. Journal of Research in Personality, 47(5), 539-546.

Lambert, N. M., Fincham, F. D., \& Stillman, T. F. (2012). Gratitude and depressive symptoms: The role of positive reframing and positive emotion. Cognition \& Emotion, 26(4), 615-633.

McCullough, M. E., Tsang, J.-A., \& Emmons, R. A. (2004). Gratitude in intermediate affective terrain: Links of grateful moods to individual differences and daily emotional experience. Journal of Personality and Social Psychology, 86(2), 295.

Ng, M.-Y., \& Wong, W.-S. (2013). The differential effects of gratitude and sleep on psychological distress in patients with chronic pain. Journal of Health Psychology, 18(2), 263-271.

Peirce, R. S., Frone, M. R., Russell, M., Cooper, M. L., \& Mudar, P. (2000). A longitudinal model of social contact, social support, depression, and alcohol use. Health Psychology, 19(1), 28.

Smith, R., Akozei, A., Killgore, W. D., \& Lane, R. D. (2018). Nested positive feedback loops in the maintenance of major depression: An integration and extension of previous models. Brain, Behavior, and Immunity, 67, 374-397.

Storch, E. A., Roberti, J. W., \& Roth, D. A. (2004). Factor structure, concurrent validity, and internal consistency of the beck depression inventory-Second edition in a sample of college students. Depression and Anxiety, 19(3), 187-189.

Toepfer, S. M., Cichy, K., \& Peters, P. (2012). Letters of gratitude: Further evidence for author benefits. Journal of Happiness Studies, 13(1), 187-201.

Watkins, P. C., Woodward, K., Stone, T., \& Kolts, R. L. (2003). Gratitude and happiness: Development of a measure of gratitude, and relationships with subjective well-being. Social Behavior and Personality: An International Journal, 31(5), 431-451.

Watkins, P. C., Uhder, J., \& Pichinevskiy, S. (2015). Grateful recounting enhances subjective well-being: The importance of grateful processing. The Journal of Positive Psychology, 10(2), 91-98.

Wood, A. M., Maltby, J., Gillett, R., Linley, P. A., \& Joseph, S. (2008). The role of gratitude in the development of social support, stress, and depression: Two longitudinal studies. Journal of Research in Personality, 42(4), 854-871.

Wood, A. M., Froh, J. J., \& Geraghty, A. W. (2010). Gratitude and well-being: A review and theoretical integration. Clinical Psychology Review, 30(7), 890-905.

Publisher's Note Springer Nature remains neutral with regard to jurisdictional claims in published maps and institutional affiliations. 